\title{
EVERY LOW BOOLEAN ALGEBRA IS ISOMORPHIC TO A RECURSIVE ONE
}

\author{
ROD DOWNEY AND CARL G. JOCKUSCH
}

(Communicated by Andreas R. Blass)

\begin{abstract}
It is shown that every (countable) Boolean algebra with a presentation of low Turing degree is isomorphic to a recursive Boolean algebra. This contrasts with a result of Feiner (1967) that there is a Boolean algebra with a presentation of degree $\leq 0^{\prime}$ which is not isomorphic to a recursive Boolean algebra. It is also shown that for each $n$ there is a finitely axiomatizable theory $T_{n}$ such that every low ${ }_{n}$ model of $T_{n}$ is isomorphic to a recursive structure but there is a low $_{n+1}$ model of $T_{n}$ which is not isomorphic to any recursive structure. In addition, we show that $n+2$ is the Turing ordinal of the same theory $T_{n}$, where, very roughly, the Turing ordinal of a theory describes the number of jumps needed to recover nontrivial information from models of the theory. These are the first known examples of theories with Turing ordinal $\alpha$ for $3 \leq \alpha<\omega$.
\end{abstract}

\section{INTRODUCTION}

The notion of a presentation of a structure is quite old and can be expressed in many ways. In "combinatorial" algebra one usually considers the complexity of the "defining object". For instance, in combinatorial group theory, a group $G$ is called finitely presented if $G$ can be expressed as the quotient of some finitely generated free group $F$ by the normal closure of a finitely generated subgroup of $F$ (see, e.g., Magnus, Karrass, and Solitar [17]). More generally, we can say a group is $\Delta_{n}^{0}$-presentable (where $\Delta_{n}^{0}$ denotes the $n$th level of Kleene's arithmetical hierarchy) if $G$ is isomorphic to a quotient of the free group on $\left\{x_{0}, x_{1}, \ldots\right\}$ by a $\Delta_{n}^{0}$ normal subgroup. There is nothing special about groups here, and many authors have studied presentations of various algebraic structures such as vector spaces, linear orderings, fields, and the like. We remark that one can easily give a logical definition of a $\Delta_{n}^{0}$ presentation of a model as one whose universe is (coded as) $\mathbb{N}$ and whose open diagram is $\triangle_{n}^{0}$.

Received by the editors November 30, 1992 and, in revised form, February 15, 1993.

1991 Mathematics Subject Classification. Primary 03C57; Secondary 03D30, 06 E99.

The first author's research is supported in part by a US/NZ Binational Grant, the Mathematical Sciences Institute at Cornell, and Cornell University. The results in this paper were established during a sabbatical year at Cornell while on a visit with the second author and supported by the NSF.

The second author was supported by NSF grant DMS 92-02833. 
In this paper our primary purpose is to solve an old question generated by the work of Feiner [7, 8] concerning presentations of Boolean algebras. Here we say a Boolean algebra $B$ is $\Delta_{n}^{0}$-(respectively, $\Sigma_{n}^{0}-, \Pi_{n}^{0}$ ) presented if $B$ is given as $\mathbf{Q} / F$ where $\mathbf{Q}$ denotes the standard countable atomless Boolean algebra and $F$ is a $\triangle_{n}^{0}$ (respectively, $\left.\Sigma_{n}^{0}, \Pi_{n}^{0}\right)$ filter of $\mathbf{Q}$.

It can be shown that every $\Pi_{1}^{0}$-presented Boolean algebra is isomorphic to a recursively presented one and that each $\Delta_{n+1}^{0}$-presented Boolean algebra is isomorphic to a $\Sigma_{n}^{0}$-presented one (Feiner; see, e.g., Downey [4]). Using a very difficult coding argument, Feiner $[7,8]$ showed that there exist $\Sigma_{n}^{0}$-presented Boolean algebras not isomorphic to $\triangle_{n}^{0}$-presented ones. In particular, there exist r.e.-presented Boolean algebras not isomorphic to recursive ones. Quite aside from its intrinsic interest, Feiner's result had some powerful consequences in classical recursion theory (such as the solution to Rogers's strong homogeneity conjecture and the result that the lattice of recursively enumerable sets is not recursively presentable) (see Feiner [7-10]).

The technique of proof used for Feiner's theorem on Boolean algebras was extremely novel and involved a special coding of $0^{(\omega)}$ into the Fréchet sequence of the r.e. Boolean algebra $B$. A consequence of the use of this or similar "high level" coding techniques is that Feiner's Boolean algebra is not even isomorphic to one with a low $_{n}$ presentation for any $n$. Here, a presentation $Q / F$ is $l o w_{n}$ if $F^{(n)}$ (the $n$th Turing jump of $F$ ) has the same degree as $0^{(n)}$. A structure is low if it is low 1 .

One can ask questions of this sort for other structures. Linear orderings tend to be the structures that are in many ways closest to Boolean algebras. For both Boolean algebras and linear orderings we know that no direct coding is possible (Richter [22, 23]). This means that if $A$ is either a linear ordering or a Boolean algebra, then there is no nonrecursive set $C$ which is recursive in every presentation of every isomorphic copy of $A$. It is, however, possible to code information into the double jump of a linear ordering. For example, for each degree $\mathbf{d} \geq 0^{\prime \prime}$ there is a linear ordering $L$ such that $\mathbf{d}$ is the least degree which is the double jump of the degree of an isomorphic copy of $L$. However, such "jump codings" are useless for showing that there is a low linear ordering not isomorphic to a recursive one. Nevertheless, Jockusch and Soare [11] used a kind of diagonal argument (rather than a coding argument) to show that there are low linear orderings not isomorphic to recursive ones. Jockusch and Soare's new technique has found several applications (see [6]).

Our main result is the following, which contrasts with the theorem mentioned above that there is a low linear ordering not isomorphic to a recursive one.

\section{Theorem 1. Every low Boolean algebra is isomorphic to a recursive one.}

The proof of Theorem 1 is surprisingly easy but does involve a new technique. This technique involves representing a given low Boolean algebra $B$ as the interval algebra of a low linear ordering $L$ and then constructing an orderpreserving $\triangle_{2}^{0}$ embedding $h$ from $L$ to a recursive linear ordering $\widehat{L}$. We should not expect to be able to make $h$ an order-isomorphism in view of the above-mentioned result that there is a low linear ordering not isomorphic to a recursive one. However, we ensure that the range of $h$ is "sufficiently large" in $\widehat{L}$ to be able to apply a theorem of Remmel [21, Theorem 1.2] to show that $L$ 
and $\widehat{L}$ have isomorphic interval algebras. Specifically, it suffices to ensure that every element of $\widehat{L}$ which is not in the range of $h$ belongs to a finite interval of $\widehat{L}$ of the form $(h(a), h(b))$.

We do not know whether, for all $n>1$, every low $_{n}$ Boolean algebra is isomorphic to a recursive one. However, in Theorem 2 we produce for each $n$ a finitely axiomatized theory $T_{n}$ such that every low $_{n}$ model of $T_{n}$ is isomorphic to a recursive one but not every low $_{n+1}$ model of $T_{n}$ is isomorphic to a recursive one. Thus, by definition, the lowness ordinal of $T_{n}$ is $n+1$. In general, a theory $T$ is said to have lowness ordinal $\alpha$, where $\alpha$ is a recursive ordinal, provided that $\alpha$ is the least ordinal $\beta$ such that $T$ has a model $A$ which is $\beta$-low (i.e., $A^{(\beta)}$ is recursive in $0^{(\beta)}$ ) and not isomorphic to any recursive structure. Note that by the result of Feiner mentioned above, there is a Boolean algebra which is $\triangle_{2}^{0}$ and hence $\omega$-low but not isomorphic to a recursive one. Thus the lowness ordinal $\lambda$ of the theory of Boolean algebras exists and (also applying Theorem 1) $2 \leq \lambda \leq \omega$.

Theorem 1 is also related to studies trying to assign a degree to the isomorphism type of a structure. Such studies explicitly began with Richter [22, 23], who defined the degree of the isomorphism type of a countable structure $A$ to be the least degree (if it exists) which occurs as the degree of the open diagram of a structure (with universe $\omega$ ) isomorphic to $A$. Thus, for example, the isomorphism type of a structure $A$ has degree 0 iff $A$ has a recursive copy. She showed that some theories $T$ (for example, the theory of abelian groups) have the property that all degrees d occur as degrees of isomorphism types of models of $T$. In the other direction, she showed that other theories $T$ have the property that no degrees $\mathbf{d}$ other than 0 occur as degrees of isomorphism types of models of $T$. Examples of the latter include the theories of linear orderings and Boolean algebras. Jockusch suggested that for structures whose isomorphism types have no degree, one might try to assign a jump degree, that is, the least degree (if it exists) which is the Turing jump of a structure in the relevant isomorphism type. Using a forcing argument, Knight [14] showed that if a linear ordering has a jump degree, this degree is $0^{\prime}$. More generally, Jockusch suggested that for recursive ordinals $\alpha$ we might try instead the $\alpha$ th jump degree, which is defined as the least degree which contains the $\alpha$ th jump of (the open diagram of) a structure in the isomorphism type. Here, the results of Ash, Jockusch, and Knight [2] together with the results of Downey and Knight [5] (see Downey [4]) establish that for each recursive ordinal $\alpha \geq 2$ and each degree $\mathbf{a} \geq 0^{(\alpha)}$ there is a linear ordering $L$ whose isomorphism type has $\alpha$ th jump degree a but does not have any $\beta$ th jump degree for any $\beta<\alpha$. Similar results have been found in settings such as, for instance, $p$-groups (Oates [19]). Jockusch and Soare [13] showed (using the machinery of Feiner [8]) that for each degree $\mathbf{d} \geq 0^{(\omega)}$ there is a Boolean algebra $B$ whose isomorphism type has $\omega$ th jump degree $\mathbf{d}$. In the other direction they showed that no Boolean algebra has an $n$th jump degree other than $0^{(n)}$ for any $n<\omega$.

These results suggest the following notion [13]. Let $\alpha$ be a recursive ordinal. A theory $T$ has Turing ordinal $\alpha$ if the following two conditions hold:

(i) For any degree $\mathbf{d} \geq 0^{(\alpha)}$ there is a model of $T$ whose isomorphism class has $\alpha$ th jump degree $d$, and

(ii) For any $\beta<\alpha$, no degree other than $0^{(\beta)}$ occurs as the $\beta$ th jump degree of any model of $T$. 
By the results mentioned above, the theories of abelian groups, linear orderings, and Boolean algebras have respective Turing ordinals 0,2 , and $\omega$. Note that if a theory has Turing ordinal $\tau$, then its lowness ordinal $\lambda$ exists and satisfies $\lambda \leq \tau+1$. (To prove this, let $\mathbf{b}$ be a degree such that $\mathbf{b}$ is low over $0^{(\tau)}$, i.e., $\mathbf{b}>0^{(\tau)}$ and $\mathbf{b}^{\prime}=0^{(\tau+1)}$, and let $A$ be a model of $T$ with $\tau$-jump degree $\mathbf{b}$. Then $A$ is $(\tau+1)$-low but not isomorphic to a recursive structure.) The following result gives examples of Turing ordinals.

Theorem 2. For each $n$ with $0 \leq n<\omega$, there is a finitely axiomatizable theory $T_{n}$ with lowness ordinal $n+1$ and Turing ordinal $n+2$.

Since it is known that there are finitely axiomatizable theories with Turing ordinals 0,1 , and $\omega$, we obtain the following corollary.

Corollary. If $0 \leq \alpha \leq \omega$, there is a finitely axiomatizable theory with Turing ordinal $\alpha$.

Notation is standard, and the reader is referred to, e.g., Soare [25] or Rogers [24]. If $L$ is a linear ordering having a least element, let $\operatorname{Intal}(L)$ (the interval algebra of $L$ ) be the Boolean algebra of sets consisting of all finite unions of left-closed, right-open intervals of $L \cup\{\infty\}$, where $\infty$ is taken to be greater than all elements of $L$.

\section{THE PROOFS}

We begin with Theorem 1. Using well-known folklore results, we can consider a low Boolean algebra as $\operatorname{Intal}(L)$, the interval subalgebra of a low linear subordering of the rationals, $\mathbb{Q}$. (See, for instance, Remmel [21, Theorem 1.2].) Recall that a pair of elements $a, b$ with $a<_{L} b$ in an ordering $L$ is called a successivity or adjacency if there is no $z \in L$ with $a<_{L} z<_{L} b$. We write $\operatorname{adj}(a, b)$ if $[a, b]$ is an adjacency. Note that the atoms of $\operatorname{Intal}(L)$ are precisely the left-closed, right-open intervals $(a, b]$ of $L \cup\{\infty\}$ such that $\operatorname{adj}(a, b)$. By Cantor's theorem, if $B=\operatorname{Intal}(L)$ and $L$ has only finitely many adjacencies, then $B$ is isomorphic to a recursive Boolean algebra. So we can suppose $L$ has infinitely many adjacencies.

Remmel [21, Theorem 2.1] obtained a surprising result related to Vaught's well-known theorem [28] that if $B$ and $\widehat{B}$ are countable atomic Boolean algebras with infinitely many atoms, and the quotient of $B$ modulo the ideal generated by its atoms is isomorphic to the corresponding quotient for $\widehat{B}$, then $B$ and $\widehat{B}$ are isomorphic. Remmel's theorem is the following: Let $B$ and $\widehat{B}$ be countable Boolean algebras such that $B$ is a subalgebra of $\widehat{B}$ and $B$ has infinitely many atoms. Suppose further that each atom of $B$ is the supremum of finitely many atoms of $\widehat{B}$, that each atom of $\widehat{B}$ lies below some atom of $B$, and that $\widehat{B}$ is generated by $B$ and the atoms of $\widehat{B}$. Then $B$ and $\widehat{B}$ are isomorphic. Applying this result to interval algebras, we obtain the following corollary, which is crucial to our proof of Theorem 1 .

Lemma 3. Let $L$ and $\widehat{L}$ be suborderings of $\mathbb{Q}$ such that $L$ has infinitely many adjacencies, and $L, \widehat{L}$ each have both a least and a greatest element. Suppose that there is a function $h: L \rightarrow \widehat{L}$ with the following properties, where $a, b$, and c range over $\mathbb{Q}$ : 
(i) $h$ is 1-1 and order preserving and maps the least [greatest] element of $L$ to the least [greatest] element of $\widehat{L}$.

(ii) If $[a, b]$ is an adjacency of $L$, then $[h(a), h(b)] \cap \widehat{L}$ is finite.

(iii) If $c \in \widehat{L}-\operatorname{range}(h)$, then $(\exists a, b)[a, b \in L \& \operatorname{Adj}(a, b) \& h(a)<c<$ $h(b)]$.

Then $\operatorname{Intal}\left(L^{-}\right) \cong \operatorname{Intal}\left(\widehat{L}^{-}\right)$, where $L^{-}, \widehat{L}^{-}$are obtained from $L, \widehat{L}$, respectively, by deleting their respective greatest elements.

In light of Lemma 3, to establish the desired result, it suffices to show that for any low linear ordering $L \subseteq \mathbb{Q}$ having least and greatest elements, there is a recursive linear ordering $\widehat{L} \subseteq \mathbb{Q}$ and a function $h: L \rightarrow \widehat{L}$ such that (i)-(iii) of Lemma 3 hold.

To construct such $\widehat{L}$ and $h$, we first note that Adj is a $\Delta_{2}^{0}$ predicate since $L$ is low. By the Limit Lemma, $L$ has a recursive approximation $L_{s}$ (so that for almost all $\left.s, a \in L \Longleftrightarrow a \in L_{s}\right)$. We may further assume that

$\operatorname{Adj}(a, b)$ implies $\left\{s:(\exists c)\left(a<c<b \& a, b, c \in L_{s}\right)\right\}$ is finite.

Using the $\Delta_{2}^{0}$-ness of Adj, we can "speed up" any approximation to $L$ to get one with this property. We may also assume that the least and greatest elements of $L$ are in $L_{0}$. We will construct $\widehat{L}=\bigcup_{s} \widehat{L}_{s}$, and $h=\lim _{s} h_{s}$ in stages using a recursive construction. At the initial stage $s=0$ we map the least [greatest] element of $L$ to 0 [1] with highest priority and never change $h$ on these arguments. At stage $s$, we are allowed to add new elements to $\widehat{L}$ between the current values of $h(a)$ and $h(b)(a<b)$ only if there exists $c \in L_{s}$ with $a<c<b$. Thus (ii) will hold.

Let $q_{0}, q_{1}, \ldots$ be a recursive enumeration of $\mathbb{Q}$. We require that any element added to $\widehat{L}$ at stage $s$ be of the form $q_{m}$ with $m \geq s$. Hence $\widehat{L}$ will be recursive.

We have the following requirements:

$$
\begin{array}{cl}
R_{2 m}: & q_{m} \in L \Longrightarrow h\left(q_{m}\right) \downarrow \& h\left(q_{m}\right) \in \widehat{L}, \\
R_{2 k+1}: & q_{k} \in \widehat{L}-h(L) \Longrightarrow(\exists i, j), \\
\operatorname{Adj}\left(q_{i}, q_{j}\right) \& h\left(q_{i}\right)<q_{k}<h\left(q_{j}\right) .
\end{array}
$$

Assign priorities as usual (the argument is finite injury). The construction is arranged so that $\operatorname{dom}\left(h_{s}\right) \subseteq L_{s}$ for every stage $s$. When we set $h_{s}\left(q_{m}\right)=q_{k}$, this assignment has the priority of $R_{p}$, where $p=\min \{2 m, 2 k+1\}$. If $q_{m} \in L_{t}$ for all $t \geq s$ and no requirement of higher priority than $R_{p}$ acts after stage $s$, we will then have $h_{t}\left(q_{m}\right)=q_{k}$ for all $t \geq s$ (and hence $\left.h\left(q_{m}\right)=q_{k}\right)$.

Strategy for $R_{2 m}$. If $q_{m} \in L_{s+1}$ and $h_{s}\left(q_{m}\right)$ is not defined, define $h_{s+1}\left(q_{m}\right)=q_{t}$ where $t$ is chosen so that $t>m$ and this definition keeps $h_{s+1}$ order preserving. Add $q_{t}$ to $\hat{L}$. If $q_{m}$ leaves $L$ or a higher priority requirement $R_{2 k+1}$ acts, cancel this value of $h$ and start over. If $q_{m} \in L$, this will happen only finitely often, and $h\left(q_{m}\right)=\lim _{s} h_{s}\left(q_{m}\right)$ will exist and be in $\widehat{L}$.

Strategy for $R_{2 k+1}$. Suppose $q_{k} \in \widehat{L}_{s}-h_{s}\left(L_{s}\right)$. (This situation arises when $q_{k}$ is put into $\widehat{L}$ by some $R_{2 m}$, but its apparent $h$-preimage seems to leave $L$ or $q_{k}$ is cancelled as an $h$-image by higher-priority action.) Further, assume that there do not exist $i$ and $j$ with $h\left(q_{i}\right)$ and $h\left(q_{j}\right)$ defined with stronger 
priority than that of $R_{2 k+1}$ such that $\operatorname{Adj}_{s}\left(q_{i}, q_{j}\right)$ and $h_{s}\left(q_{i}\right)<q_{k}<h_{s}\left(q_{j}\right)$. Cancel all lower-priority values of $h$. Choose $i$ and $j$ with $h_{s}\left(q_{i}\right)$ and $h_{s}\left(q_{j}\right)$ defined with stronger priority than $R_{2 k+1}$ such that $h\left(q_{i}\right)<q_{k}<h\left(q_{j}\right)$ and $\left(h\left(q_{i}\right), h\left(q_{j}\right)\right)$ contains no values of $h$ defined with stronger priority than that of $R_{2 k+1}$. (Such $i$ and $j$ exist because we initially defined $h$ on the least and greatest elements of $L$ with highest priority.) By hypothesis, $\operatorname{Adj}_{s}\left(q_{i}, q_{j}\right)$ does not hold. As long as it continues not to hold, search for $t$ such that $q_{t} \in L$ and $q_{i}<q_{t}<q_{j}$ by recursive approximation. Set $h\left(q_{t}\right)=q_{k}$ (without changing $\widehat{L}$ ). If the candidate for $t$ changes, start over by undefining all lower-priority values of $h$. Also, if $\operatorname{Adj}\left(q_{i}, q_{j}\right)$ starts to hold or $q_{i}$ or $q_{j}$ appears to leave $L$, start over.

These strategies combine by a standard finite injury argument. We omit further details, which are routine. Observe that the argument actually shows that any $\triangle_{2}^{0}$-presented Boolean algebra with a $\Delta_{2}^{0}$ set of atoms is isomorphic to a recursive Boolean algebra.

We remark that it seems a very interesting open question whether the result above is sharp in terms of the high/low hierarchy. For instance, is it true for every $n$ that every low $_{n}$ Boolean algebra $B$ is isomorphic to a recursive one? \{Added remark (August 1993): A positive answer for $n=2$ has recently been obtained by Thurber [27]. Thus the lowness ordinal $\lambda$ of the theory of Boolean algebras satisfies $3 \leq \lambda \leq \omega$, although its exact value remains unknown. Thurber's proof uses Remmel's isomorphism theorem [21, Theorem 2.1] and our observation in the previous paragraph.\} This question is related to the results of Jockusch and Soare [12], who show that if a Boolean algebra has $n$th jump degree and $n<\omega$, then that degree is $0^{(n)}$. If, for all $n$, all low $_{n}$ Boolean algebras are isomorphic to recursive ones, then (combining this with the Jockusch-Soare result just mentioned) every Boolean algebra having an $n$th jump degree is trivial in the sense that it has a recursive copy. On the other hand, the Jockusch-Soare result alone is consistent with the existence of a properly low $n$ Boolean algebra. (Compare with the facts that if a linear ordering has jump degree, it is $0^{\prime}$ (Knight [14]), yet there exist low linear orderings not isomorphic to recursive ones (Jockusch and Soare [12]).)

This brings us to the proof of Theorem 2. We must show that for each $n$ there is a finitely axiomatizable theory $T_{n}$ with lowness ordinal $n+1$ and Turing ordinal $n+2$.

To start the proof of Theorem 2, let $T_{0}$ be the theory of linear order. The existence of a low linear ordering not isomorphic to a recursive one [12] implies that the lowness ordinal of $T_{0}$ is 1 . By Knight [14], no model of $T_{0}$ has a first jump degree other than $0^{\prime}$. By Downey and Knight [5], every degree $\mathbf{d} \geq 0^{\prime \prime}$ is the second jump degree of a model of $T_{0}$. (The latter may also be easily proved using the first four lines of the proof of Theorem 2.4 of Ash, Jockusch, and Knight [2], as we do not require that the orderings involved have no first jump degree.) Thus the Turing ordinal of $T_{0}$ is 2 .

As in [2], for an ordering $L$, let

$$
D g(L)=\{\operatorname{deg}(D): D \cong L\}
$$

The general case is based on the $n=0$ case together with a further crucial use of an ordering from Downey and Knight [5] to "push up" the number of 
jumps involved. For a linear ordering $L$, let

$$
\varphi(L)=(\eta+2+\eta) \cdot L
$$

where $\eta$ denotes the order type of the rationals. That is, each point of $L$ is replaced by $\eta+2+\eta$.

To obtain the desired theories $T_{n}$, we next observe that for each $n$ there is a finitely axiomatizable theory $T_{n}$ such that the countable models of $T_{n}$ are exactly the linear orderings which, for some countable linear ordering $L$, are isomorphic to $\varphi^{n}(L)$. As mentioned, we simply take $T_{0}$ to be the theory of linear order. For $T_{1}$, the axioms are those of $T_{0}$ together with axioms stating that for all $d$, if $d$ is not part of an adjacency, then either there is an adjacency $[a, b]$ such that $(b, d)$ is dense or an adjacency $[a, b]$ such that $(d, a)$ is dense. Proceeding by induction, for $m \geq 1$ the axioms of $T_{m+1}$ are those of $T_{m}$ together with axioms asserting that the adjacencies obey the axioms of $T_{m}$.

As in [2], for an ordering $L$, let

$$
D g(L)=\{\operatorname{deg}(D): D \cong L\} .
$$

In Downey and Knight [5], it is shown that $L$ is presentable in degree $d^{\prime}$ iff $\varphi(L)$ is presentable in degree d. Let

$$
\varphi^{n}(L)=\varphi(\varphi \cdots \varphi(L) \cdots) \quad(n \text { times }) .
$$

It follows immediately by induction, as observed in Downey and Knight [5], that

$$
D g\left(\varphi^{n}(L)\right)=\left\{\mathbf{d}: \mathbf{d}^{(n)} \in D g(L)\right\} .
$$

This is the "pushing up" phenomenon that we need.

We now show that $T_{n}$ has lowness ordinal $n+1$. First we show that any low $_{n}$ model of $T_{n}$ is isomorphic to a recursive structure. It suffices to show that if $L$ is a linear ordering such that $D g(L)$ contains a degree d such that $\mathbf{d}^{(n)}=0^{(n)}$, then $L$ is isomorphic to a recursive structure. This is clear from $(*)$, which shows that membership of a degree in $D g\left(\varphi^{n}(L)\right)$ depends only on the $n$th jump of the degree. Thus the lowness ordinal of $T_{n}$ is at least $n+1$ if it exists. To show that this ordinal is exactly $n+1$, we must produce a low $_{n+1}$ model of $T_{n}$ not isomorphic to a recursive one. By relativising the proof of existence of a low linear ordering not isomorphic to a recursive one [12] to $0^{(n)}$, one may show that there is a degree $\mathbf{b}$ which is low over $0^{(n)}$ (i.e., $\mathbf{b}>0^{(n)}$ and $\left.\mathbf{b}^{\prime}=0^{(n+1)}\right)$ ) and an ordering $L$ such that $\mathbf{b} \in D g(L)$ and $0^{(n)} \notin D g(L)$. By iterated jump inversion [26, Corollary VI.3.2], there is a degree $\mathbf{c}$ such that $\mathbf{c}^{(n)}=\mathbf{b}$. By $(*), \mathbf{c} \in D g\left(\varphi^{n}(L)\right)$. Since $\mathbf{c}$ is $\operatorname{low}_{n+1}$, there is a low $_{n+1}$ ordering

$$
\widehat{L} \cong \varphi^{n}(L) .
$$

If $\widehat{L}$ were isomorphic to a recursive ordering, we would have $0^{(n)} \in D g(L)$ by $(*)$, contrary to our choice of $L$. This shows that we may take $\widehat{L}$ as our desired low $w_{n+1}$ model of $T_{n}$ not isomorphic to a recursive one. This completes the proof that $T_{n}$ has lowness ordinal $n+1$.

Next, we show that the Turing ordinal of $T_{n}$ is $n+2$. We will use the following consequence of $(*)$ :

$$
\left\{\mathbf{d}^{(n+1)}: \mathbf{d} \in D g\left(\varphi^{n}(L)\right\}=\left\{\mathbf{e}^{\prime}: \mathbf{e} \geq 0^{(n)} \& \mathbf{e} \in D g(L)\right\} .\right.
$$


The above follows at once from $(*)$ and iterated jump inversion (i.e., let $\left.e=d^{(n)}\right)$. By relativising to $0^{(n)}$ the proof of Knight's result [14, Corollary 3.6] that $0^{\prime}$ is the only possible first jump degree for an ordering, one may show that if $L$ is a linear ordering and $\mathrm{c}$ is the least element of $\left\{\mathbf{e}^{\prime}: \mathbf{e} \geq 0^{(n)} \& \mathbf{e} \in D g(L)\right\}$ then $\mathbf{c}=0^{(n+1)}$. Thus, $(* *)$ implies that if $\mathbf{c}$ is the $(n+1)$ st jump degree of $\varphi^{n}(L)$ then $\mathrm{c}=0^{(n+1)}$.

Finally we show that for any degree $c \geq 0^{(n+2)}$ there is a linear ordering $L$ such that $\mathrm{c}$ is the $(n+2)$ nd jump degree of $\varphi^{n}(L)$. Given such a $\mathrm{c}$, by iterated, relativised jump inversion there is a degree $\mathbf{a} \geq 0^{\prime \prime}$ such that $\mathbf{c}=\mathbf{a}^{(n)}=$ a $\cup 0^{(n+2)}$. By the case $n=0$ of our theorem there is a linear ordering $L$ which has second jump degree a. To show that $\varphi^{n}(L)$ has $(n+2)$ nd jump degree c, we show first that there is a degree $\mathbf{b} \in D g\left(\varphi^{n}(L)\right)$ such that $\mathbf{b}^{(n+2)}=\mathbf{c}$. For this, choose $\mathbf{b} \in D g(L)$ with $\mathbf{b}^{\prime \prime}=\mathbf{a}$. Then, since $D g(L) \subseteq D g\left(\varphi^{n}(L)\right)$, $\mathbf{b} \in D g\left(\varphi^{n}(L)\right)$, and clearly $\mathbf{b}^{(n+2)}=\mathbf{a}^{(n)}=\mathbf{c}$, as required. Second, we must show that if $\mathbf{d} \in D g\left(\varphi^{n}(L)\right)$ then $\mathbf{d}^{(n+2)} \geq \mathbf{c}$. Now $\mathbf{d}^{(n)} \in D g(L)$ by $(*)$, so $\mathbf{d}^{(n+2)} \geq \mathbf{a}$ since $L$ has second jump degree $\mathbf{a}$. Therefore, $\mathbf{d}^{(n+2)} \geq \mathbf{a} \cup 0^{(n+2)}=\mathbf{c}$, as required.

Corollary. For each $\alpha$ with $0 \leq \alpha \leq \omega$ there is a finitely axiomatized theory with Turing ordinal $\alpha$.

As remarked in the introduction, the theory of abelian groups has Turing ordinal 0 [23] and the theory of Boolean algebras has Turing ordinal $\omega$ [13]. Thus, to conclude the proof of the corollary, it suffices to show that there is a finitely axiomatized theory with Turing ordinal 1. Several examples of such theories are known. For instance, Knight [14, remarks, pp. 1034-1035] establishes that Peano Arithmetic has Turing ordinal 1 , and her argument easily adapts to show that there is a finitely axiomatized fragment of Peano arithmetic which has Turing ordinal 1 . This is perhaps the most accessible and interesting example. Also Richter, in her thesis [22, Theorems 10.1 and 13.1] proved that the theory of trees (in the language of partial orderings) also has Turing ordinal 1. For further examples we can again use her thesis [22, Theorems 10.2 and 11.2]. In these it is shown that if $T$ is either the theory of an equivalence relation or the theory of ordering with a predicate for adjacency, no model of $T$ can have a degree other than 0 . It is easy to complete the proof that these theories have Turing ordinal 1 by constructing for each degree $\mathbf{d} \geq 0^{\prime}$ a model with first jump degree d.

We remark that it is easy to show from the proof of Theorem 2 and a known result that the theory of discrete linear orderings has lowness ordinal 3 and Turing ordinal 4 . Let $T_{\text {dis }}$ be the theory of discrete linear orderings, i.e., linear orderings in which each element except the least has an immediate predecessor and each element not the greatest has an immediate successor. Then each model of $T_{\text {dis }}$ is either finite or is isomorphic to an ordering of the form $\omega+$ $\left(\omega^{*}+\omega\right) \cdot L+\omega^{*}$, where $L$ may be empty, or to the result of omitting the initial $\omega$ or the final $\omega^{*}$ from such an ordering. We use the notation of the proof of Theorem 2. Watnick [29] showed in essence that, for a countable linear ordering $L$,

$$
D g\left(\left(\omega^{*}+\omega\right) \cdot L\right)=\left\{\mathbf{d}^{\prime \prime}: \mathbf{d} \in D g(L)\right\} \text {. }
$$

(See [2, Lemma 2.2], where the result was rediscovered, for a different proof.) 
Thus, by $(*)$,

$$
D g\left(\left(\omega^{*}+\omega\right) \cdot L\right)=D g\left(\varphi^{2}(L)\right) \text {. }
$$

It follows that for any countable model $L_{1}$ of $T_{\text {dis }}$ there is a countable model $L_{2}$ of $T_{2}$ such that $D g\left(L_{1}\right)=D g\left(L_{2}\right)$, and vice-versa. Hence $T_{2}$ and $T_{\text {dis }}$ have the same Turing and lowness ordinals. Therefore, by Theorem $2, T_{\text {dis }}$ has lowness ordinal 3 and Turing ordinal 4 .

\section{REFERENCES}

1. C. J. Ash, Stability of recursive structures in the arithmetical degrees, Ann. Pure Appl. Logic 32 (1986), 113-135.

2. C. J. Ash, C. Jockusch, and J. F. Knight, Jumps of orderings, Trans. Amer. Math. Soc. 319 (1991), 573-599.

3. R. G. Downey, Every recursive Boolean algebra is isomorphic to one with incomplete atoms, Ann. Pure Appl. Logic 60 (1993), 193-206.

4. __ Recursion theory and linear orderings, Handbook of Recursive Algebra (A. Nerode and J. Remmel, eds.) (to appear).

5. R. G. Downey and J. F. Knight, Orderings with $\alpha$ th jump degree $0^{(\alpha)}$, Proc. Amer. Math. Soc. 114 (1992), 545-552.

6. R. G. Downey and M. F. Moses, Recursive linear orderings with incomplete successivities, Trans. Amer. Math. Soc. 326 (1991), 653-668.

7. L. J. Feiner, Orderings and Boolean algebras not isomorphic to recursive ones, Thesis, MIT, Cambridge, MA, 1967.

8. __ Hierarchies of Boolean algebras, J. Symbolic Logic 35 (1970), 365-374.

9. __ The strong homogeneity conjecture, J. Symbolic Logic 35 (1970), 375-377.

10. _ Degrees of non-recursive presentability, Proc. Amer. Math. Soc. 38 (1973), 621-624.

11. S. S. Goncharov, Some properties of the constructivization of Boolean algebras, Sibirski Mat. Zh. 16 (1975), 264-278.

12. C. G. Jockusch and R. I. Soare, Degrees of orderings not isomorphic to recursive linear orderings, Ann. Pure Appl. Logic 52 (1991), 39-64.

13. C. G. Jockusch and R. I. Soare, Boolean algebras, Stone spaces, and the iterated Turing jump, preprint.

14. J. F. Knight, Degrees coded in jumps of orderings, J. Symbolic Logic 51 (1986), 1034-1042.

15. __ A metatheorem for finitely many workers, J. Symbolic Logic 55 (1990), 787-804.

16. M. Lerman, On recursive linear orders, Logic Year 1979-80 (Lerman, Schmerl, and Soare, eds.), Lecture Notes in Math., vol. 859, Springer-Verlag, New York, 1981, pp. 132-142.

17. W. Magnus, A. Karrass, and D. Solitar, Combinatorial group theory, Academic Press, New York, 1965.

18. D. Monk, Handbook of Boolean algebras, Vol. 3, North-Holland, Amsterdam, 1989.

19. S. Oates, Jump degrees of groups, Ph.D. thesis, Univ. of Notre Dame, 1989.

20. J. B. Remmel, Recursive Boolean algebras with recursive atoms, J. Symbolic Logic 46 (1981), 595-616.

21. _ Recursive isomorphism types of recursive Boolean algebras, J. Symbolic Logic 46 (1981), 572-594.

22. L. J. Richter, Degrees of unsolvability of models, Ph.D. thesis, University of Illinois at Urbana-Champaign, 1977.

23. __ Degrees of structures, J. Symbolic Logic 46 (1981), 723-731.

24. H. J. Rogers, Theory of recursive functions and effective computability, McGraw-Hill, New York, 1967. 
25. J. Rosenstein, Linear orderings, Academic Press, New York, 1982.

26. R. I. Soare, Recursively enumerable sets and degrees, Springer-Verlag, New York, 1987.

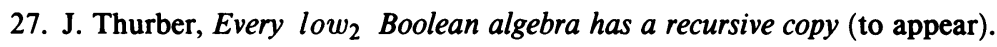

28. R. Vaught, Topics in the theory of arithmetical classes and Boolean algebras, Ph.D. thesis, University of California, Berkeley, 1954.

29. R. Watnick, A generalization of Tennenbaum's theorem on effectively finite linear orderings, J. Symbolic Logic 49 (1984), 563-569.

Department of Mathematics, Cornell University, Ithaca, New York 14853

Current address: Department of Mathematics, Victoria University, P.O. Box 600, Wellington, New Zealand

E-mail address: downey@math. vuw.ac.nz

Department of Mathematics, University of Illinois, 1409 West Green Street, Urbana, ILLINOIS 61801

E-mail address: jockusch@math.uiuc.edu 